\title{
A WEIGHTED $H$ (div) LEAST-SQUARES METHOD FOR SECOND-ORDER ELLIPTIC PROBLEMS
}

\author{
Z. CAI* ${ }^{*}$ AND C. R. WESTPHAL ${ }^{\dagger \ddagger}$
}

\begin{abstract}
This paper presents analysis of a weighted-norm least squares finite element method for elliptic problems with boundary singularities. We use $H($ div $)$ conforming Raviart-Thomas elements and continuous piecewise polynomial elements. With only a rough estimate of the power of the singularity, we employ a simple, locally weighted $L^{2}$ norm to eliminate the pollution effect and recover better rates of convergence. Theoretical results are carried out in weighted Sobolev spaces and include ellipticity bounds of the homogeneous least-squares functional, new weighted Raviart-Thomas interpolation results, and error estimates in both weighted and non-weighted norms. Numerical tests are given to confirm the theoretical estimates and to illustrate the practicality of the method
\end{abstract}

Key words. boundary singularities, least squares, finite element methods, weighted Sobolev spaces

AMS subject classifications. 65N30, 65N12, 35B65

1. Introduction. The loss of regularity of second-order partial differential equations at corners/edges of polygonal/polyhedral domains is well-known. Numerical methods for approximating solutions to these problems often suffer either reduced rates of accuracy or loss of convergence due to the nonsmooth solutions. For finite element methods, the rate of convergence generally depends on both the smoothness of the solution and the approximation properties of the finite element space used. When the rates are diminished due to these boundary singularities, standard rates of convergence can only be recovered with care.

In this paper we present a weighted-norm least-squares method for scalar elliptic problems of the form:

$$
\left\{\begin{aligned}
-\nabla \cdot A \nabla u+\mathbf{b} \cdot \nabla u+c u & =f & & \text { in } \Omega, \\
u & =0 & & \text { on } \partial \Omega,
\end{aligned}\right.
$$

where $A$ is symmetric, positive definite and $\mathbf{b}$ and $c$ are smooth, bounded functions. For the analysis in this paper we assume homogenous Dirichlet boundary conditions, though the method may easily be applied to other boundary conditions. It is wellknown that if $\Omega$ is a polygonal/polyhedral domain in $\mathbb{R}^{2} / \mathbb{R}^{3}$ then the regularity of system (1.1) may be limited by the geometry of $\Omega$. To illustrate, assume that $\Omega \subseteq \mathbb{R}^{2}$ has one reentrant corner, which has interior angle $\omega$, and $(r, \theta)$ are polar coordinates centered at the corner. For $f \in L^{2}(\Omega)$, solutions to (1.1) may be decomposed by

$$
u=\tilde{u}+r^{\lambda} w
$$

where $\tilde{u} \in H^{2}(\Omega)$ is the smooth component of $u$, and $r^{\lambda} w \in H^{\alpha+1}(\Omega)$ for $\alpha<\lambda$ is the singular component. The function $w=w(\theta)$ and parameter $\alpha$ are characteristic of the operator and the domain. For Poisson's equation the singular functions are multiples of $r^{\pi / \omega} \sin (\pi \theta / \omega)$. Edge singularities in $\mathbb{R}^{3}$ may be considered analogously, where $w=w(\theta, z)$.

\footnotetext{
*Department of Mathematics, Purdue University; West Lafayette, IN, USA. Email: zcai@math. purdue.edu

${ }^{\dagger}$ Department of Mathematics and Computer Science, Wabash College, PO Box 352, Crawfordsville, IN 47933, USA. Email: westphac@wabash.edu.

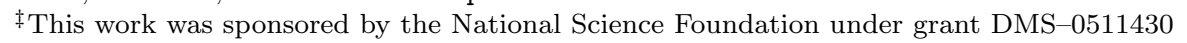


The least-squares finite element approach for a variety of problems affords several unique features. Among these is the freedom to independently choose finite element spaces for different unknowns. In [5], system (1.1) is reformulated as a first-order system by introducing the flux variable $\boldsymbol{\sigma}=-A \nabla u$, and $(u, \boldsymbol{\sigma}) \in H^{1} \times H($ div $)$ are approximated using piecewise polynomial and $H($ div $)$-conforming Raviart-Thomas elements. See also $[4,9]$ for results concerning least-squares minimization of this system. For polygonal/polyhedral domains this method results in $O\left(h^{\alpha}\right)$ approximations, even for smooth data or higher-order approximation spaces. Thus, for problems with $\alpha<1$, the convergence is suboptimal. Moreover, the reduction in convergence rate is generally a global effect-that is, convergence throughout the entire domain is suboptimal. This well-known pollution effect is also seen in Galerkin discretizations as well as div-curl least-squares formulations. We overcome this difficulty by minimizing an appropriately weighted functional. Our approach eliminates the pollution effect with only a trivial modification of the equations, requires only crude a priori knowledge of the power of the singularity (not the singular function itself), and can be used in conjunction with adaptive mesh refinement. A least-squares formulation provides a natural error estimator that can be used in an adaptive mesh refinement strategy. We note that, while many problems are effectively treated with only mesh refinement near boundary singularities, some problems admit solutions much less smooth than considered here. If the approximation space used is not dense in the solution space, then global errors may persist, despite any amount of mesh refinement. In these cases, a weighted-norm method in the spirit of this paper can be effectively used to recover convergence, allowing mesh refinement to be much less aggressive.

In [6], Cox and Fix use a weighted norm approach similar to our approach, but achieve optimal convergence with continuous $P_{1}$ finite elements and specialized graded meshes. In $[10,11]$ the weighted norm least squares approach is presented for divcurl functionals in 2-d and 3-d. An aggressive weighting is used to recover optimal convergence without relying on mesh refinement. In this paper, using mixed affine finite element spaces allows a less-aggressive weighting to achieve optimal results, even with uniform meshes.

The organization of this paper is as follows. We define notation and detail the problem formulation in section 2 . In section 3 , we show equivalence of the homogeneous least-squares functional to an appropriate weighted norm, present new interpolation bounds for Raviart-Thomas finite element spaces in weighted norms and show the resulting error bounds. Numerical results are given in section 4 that confirm the theoretical error estimates and demonstrate the practicality of the method.

2. Problem Formulation. We use standard notation for the $L^{2}$ norm, $\|\cdot\|$, and inner product, $\langle\cdot, \cdot\rangle$, and use $\|\cdot\|_{k ; \Omega}$ to denote the norm corresponding to the Sobolev space $H^{k}(\Omega)^{d}$, omitting subscript $\Omega$ and superscript $d$ when the domain and dimension are clear by context. For noninteger $k, H^{k}(\Omega)$ represents the standard interpolation space.

For this paper, we assume that $\Omega$ is a bounded domain in $\mathbb{R}^{d}, d=2,3$, with one boundary corner in $\mathbb{R}^{2}$ or one boundary edge in $\mathbb{R}^{3}$ that admits a singularity with $\alpha<1$. Assume further that this singular point is located at the origin for $d=2$ or along the $z$-axis for $d=3$, and denote this part of $\partial \Omega$ by $\mathcal{E}$. For simplicity, we consider only one singular point although techniques here may be applied locally to more than one singular point. In section 4 we discuss practical implementation in such cases. 
Define the weighted Sobolev norm by

$$
\|v\|_{k, \beta ; \Omega}=\left(\sum_{|j| \leq k}\left\|r^{\beta-k+|j|} D^{j} v\right\|_{0 ; \Omega}^{2}\right)^{1 / 2}
$$

and semi-norm by

$$
|v|_{k, \beta ; \Omega}=\left(\sum_{|j|=k}\left\|r^{\beta} D^{j} v\right\|_{0 ; \Omega}^{2}\right)^{1 / 2}
$$

For $k=0,1$, we have

$$
\begin{gathered}
\|v\|_{0, \beta ; \Omega}=\left\|r^{\beta} v\right\|_{0 ; \Omega}, \quad\|v\|_{1, \beta ; \Omega}=\left(\left\|r^{\beta} \nabla v\right\|_{0 ; \Omega}^{2}+\left\|r^{\beta-1} v\right\|_{0 ; \Omega}^{2}\right)^{1 / 2} \\
\text { and } \quad|v|_{1, \beta ; \Omega}=\left\|r^{\beta} \nabla v\right\|_{0 ; \Omega} .
\end{gathered}
$$

Then the weighted Sobolev space $H_{\beta}^{k}(\Omega)$ is defined by

$$
H_{\beta}^{k}(\Omega)=\left\{v \in L^{2}(\Omega):\|v\|_{k, \beta ; \Omega}<+\infty\right\} .
$$

The dual spaces are defined by duality in the natural way. Let

$$
D_{-\beta}^{1}=\left\{v \in H_{-\beta}^{1}(\Omega): v=0 \text { on } \partial \Omega\right\},
$$

and define its dual, $H_{\beta}^{-1}(\Omega)$, by the norm

$$
\|v\|_{-1, \beta}=\sup _{\phi \in D_{-\beta}^{1}} \frac{\langle v, \phi\rangle}{\|\phi\|_{1,-\beta}} .
$$

By introducing the new unknown $\sigma=-A \nabla u$ and denoting the lower order terms by $X u=\mathbf{b} \cdot \nabla u+c u$, we may expand system (1.1) to the first-order system

$$
\left\{\begin{aligned}
\boldsymbol{\sigma}+A \nabla u=0 & & \text { in } \Omega \\
\nabla \cdot \boldsymbol{\sigma}+X u=f & & \text { in } \Omega \\
u=0 & & \text { on } \partial \Omega .
\end{aligned}\right.
$$

In this paper, we consider the weighted least-squares functional

$$
G(u, \boldsymbol{\sigma} ; f)=\left\|A^{-1 / 2}(\boldsymbol{\sigma}+A \nabla u)\right\|_{0, \beta_{1}}^{2}+\|\nabla \cdot \boldsymbol{\sigma}+X u-f\|_{0, \beta_{2}}^{2}
$$

for functions $(u, \boldsymbol{\sigma}) \in \mathcal{V} \times \mathcal{W}$, where

$$
\mathcal{V}=\left\{v \in H_{\beta_{1}}^{1}(\Omega): v=0 \text { on } \partial \Omega\right\}
$$

and

$$
\mathcal{W}=\left\{\boldsymbol{\tau}: r^{\beta_{1}} \boldsymbol{\tau} \in L^{2}(\Omega), r^{\beta_{2}} \nabla \cdot \boldsymbol{\tau} \in L^{2}(\Omega)\right\} .
$$

The non-weighted functional (i.e., $\beta_{1}=\beta_{2}=0$ ) yields less-than-optimal global discretization rates for problems with $\alpha<1$. We thus investigate appropriate values for $\beta_{1}$ and $\beta_{2}$ to recover better rates of convergence and eliminate the pollution effect. 
The least-squares minimization problem is to find $(u, \sigma) \in \mathcal{V} \times \mathcal{W}$ such that

$$
G(u, \boldsymbol{\sigma} ; f)=\inf _{(v, \boldsymbol{\tau}) \in \mathcal{V} \times \mathcal{W}} G(v, \boldsymbol{\tau} ; f) .
$$

It may be noted that the minimization above is equivalent to the following symmetric variational problem of finding $(u, \sigma) \in \mathcal{V} \times \mathcal{W}$ such that

$$
b(u, \boldsymbol{\sigma} ; v, \boldsymbol{\tau})=f(v, \boldsymbol{\tau}) \quad \text { for all }(v, \boldsymbol{\tau}) \in \mathcal{V} \times \mathcal{W},
$$

where the bilinear and linear forms are given by

$$
b(u, \boldsymbol{\sigma} ; v, \boldsymbol{\tau}):=\left\langle r^{2 \beta_{1}} A^{-1}(\boldsymbol{\sigma}+A \nabla u), \boldsymbol{\tau}+A \nabla v\right\rangle+\left\langle r^{2 \beta_{2}}(\nabla \cdot \boldsymbol{\sigma}+X u), \nabla \cdot \boldsymbol{\tau}+X v\right\rangle
$$

and

$$
f(v, \boldsymbol{\tau}):=\left\langle r^{2 \beta_{2}} f, \nabla \cdot \boldsymbol{\tau}+X v\right\rangle
$$

Let $\mathcal{T}_{h}$ be a regular affine triangulation of $\Omega$ with a meshsize of $O(h)$, where on element $K$ we denote $h_{K}=\operatorname{diam}(K)$. Denote by $P_{k}(K)$ the standard space of polynomials of degree $\leq k$ on element $K$, and consider the Raviart-Thomas space of degree $k$ on element $K$ defined by

$$
R T_{k}(K)=P_{k}(K)^{d}+\mathbf{x} P_{k}(K)
$$

where $\mathbf{x}=\left(x_{1}, \ldots, x_{d}\right)^{t}$. For this paper we define the spaces

$$
\begin{aligned}
\mathcal{V}^{h} & =\left\{v \in \mathcal{V} \cap C^{0}(\Omega):\left.v\right|_{K} \in P_{1}(K) \forall K \in \mathcal{T}_{h}\right\}, \\
\mathcal{W}^{h} & =\left\{\boldsymbol{\tau} \in \mathcal{W}:\left.\boldsymbol{\tau}\right|_{K} \in R T_{0}(K) \forall K \in \mathcal{T}_{h}\right\} .
\end{aligned}
$$

The discrete minimization problem is to find $\left(u^{h}, \sigma^{h}\right) \in \mathcal{V}^{h} \times \mathcal{W}^{h}$ such that

$$
G\left(u^{h}, \boldsymbol{\sigma}^{h} ; f\right)=\min _{\left(v^{h}, \boldsymbol{\tau}^{h}\right) \in \mathcal{V}^{h} \times \mathcal{W}^{h}} G\left(v^{h}, \boldsymbol{\tau}^{h} ; f\right) .
$$

This leads to a symmetric, positive definite linear system of equations that can be efficiently solved by standard multigrid methods.

3. Theory. In the following analysis, we assume that $A$ is a $d \times d$ matrix of functions in $L^{2}(\Omega)$ and that $\mathbf{b}$ and $c$ are smooth, bounded functions. We further assume that $A$ is uniformly symmetric positive definite, that is, there exist positive constants $\lambda_{1}$ and $\lambda_{2}$ such that

$$
\lambda_{1} \boldsymbol{\xi}^{T} \boldsymbol{\xi} \leq \boldsymbol{\xi}^{T} A \boldsymbol{\xi} \leq \lambda_{2} \boldsymbol{\xi}^{T} \boldsymbol{\xi}
$$

for all $\boldsymbol{\xi} \in \mathbb{R}^{d}$ and almost all $\boldsymbol{\xi} \in \bar{\Omega}$. Under an ellipticity assumption on the operator for problem (1.1), a general regularity result for second-order problems is established in [1]. We state here an $H_{\beta}^{1}$ estimate sufficient for our purposes.

Theorem 3.1 Assume that the operator $L:=-\nabla \cdot A \nabla+X$ satisfies the assumptions of Theorem 5.2 in [1] and that $f \in H_{\beta}^{-1}(\Omega)$. Then there exists some $\alpha>0$ such that problem (1.1) admits the estimate

$$
\|u\|_{1, \beta} \leq C\|f\|_{-1, \beta}
$$

for any $|\beta|<\alpha$. In general, $\alpha$ depends on the operator and domain. If $L=-\Delta$, then $\alpha=\pi / \omega$, where $\omega$ is the interior angle at $\mathcal{E}$. 
Proof. See Theorem 5.4 in [1].

We note that values for $\alpha$ are considered in the classic works [7, 8]. In [12], values for $\alpha$ are explicitly shown to satisfy $\alpha \geq 1 / 2$ for the Stokes and elasticity systems. In general, the value of $\alpha$ for Poisson's equation serves as an upper bound for secondorder problems on similar geometries. While the method described in this paper is based on the value of $\alpha$ for the operator of the problem, we note that the exact value of $\alpha$ need not be known for the method to be effective.

Let $\mathcal{I}^{h}$ be the standard interpolation operator onto $P_{k}(K)$, where, for all $v \in$ $H^{m}(K)$,

$$
\left\|v-\mathcal{I}^{h} v\right\|_{s ; K} \leq C h_{K}^{m-s}|v|_{m ; K}
$$

holds for $0 \leq s \leq m$ and $1<m \leq k+1$. A proof of this classical result can be found in $[2]$.

Define the modified interpolation operator, $\mathcal{I}_{0}^{h}$, by

$$
\left.\mathcal{I}_{0}^{h} v\right|_{K}=\left\{\begin{aligned}
\left.\mathcal{I}^{h} v\right|_{K}, & \text { if } \bar{K} \cap \mathcal{E}=\emptyset, \\
\sum_{a_{i} \notin \mathcal{E}} v\left(a_{i}\right) \phi_{i}, & \text { if } \bar{K} \cap \mathcal{E} \neq \emptyset,
\end{aligned}\right.
$$

where $a_{i}$ are the nodal points corresponding to the basis functions, $\phi_{i}$. Thus, the modified interpolation has a value of zero at $\mathcal{E}$ and is identical to $\mathcal{I}^{h}$ away from $\mathcal{E}$. From $[10,11]$, if $\beta>0$ then $\mathcal{I}_{0}^{h}$ satisfies

$$
\sum_{K \in \mathcal{T}_{h}}\left\|v-\mathcal{I}_{0}^{h} v\right\|_{1, \beta ; K}^{2} \leq C h^{2}\|v\|_{2, \beta}^{2}
$$

for $v \in H_{\beta}^{2}(\Omega)$.

Let $I^{h}$ be the standard interpolation operator from $H($ div $) \cap L^{p}(\Omega)$ onto $R T_{k}$ with $p>2$. Then the following approximation properties of $R T_{k}$ hold (see, e.g., [3]) for any $\boldsymbol{\tau} \in\left(H^{m}(K)\right)^{d}$ with $1 \leq m \leq k+1$,

$$
\left\|\boldsymbol{\tau}-I^{h} \boldsymbol{\tau}\right\|_{s ; K} \leq C h_{K}^{m-s}|\boldsymbol{\tau}|_{m ; K} \quad \text { with } \quad s=0,1,
$$

and

$$
\left\|\nabla \cdot\left(\boldsymbol{\tau}-I^{h} \boldsymbol{\tau}\right)\right\|_{0 ; K} \leq C h_{K}^{m}|\nabla \cdot \boldsymbol{\tau}|_{m ; K}
$$

if $\nabla \cdot \boldsymbol{\tau} \in H^{m}(K)$. For any $\boldsymbol{\tau} \in\left(H^{\alpha}(K)\right)^{d}$ and $\nabla \cdot \boldsymbol{\tau} \in H^{\alpha}(K)$ with $\alpha<1$, we assume the following approximation properties:

$$
\left\|\boldsymbol{\tau}-I^{h} \boldsymbol{\tau}\right\| \leq C h^{\tilde{\alpha}}|\boldsymbol{\tau}|_{\alpha}
$$

and

$$
\left\|\nabla \cdot\left(\boldsymbol{\tau}-I^{h} \boldsymbol{\tau}\right)\right\| \leq C h^{\tilde{\alpha}}|\nabla \cdot \boldsymbol{\tau}|_{\alpha}
$$

hold for some constant $\tilde{\alpha} \in(0, \alpha]$.

Theorem 3.2 Assume that $\nabla \cdot \boldsymbol{\tau}$ and each $\boldsymbol{\tau}_{j}$ are in $H_{\beta}^{1}(\Omega) \cap H^{\alpha}(\Omega)$. Then for any $\beta \geq 0$, there exists a positive constant $C$ such that

$$
\left\|\boldsymbol{\tau}-I^{h} \boldsymbol{\tau}\right\|_{0, \beta} \leq C h^{m_{1}}\left(|\boldsymbol{\tau}|_{1, \beta}+|\boldsymbol{\tau}|_{\alpha}\right)
$$


and that

$$
\left\|\nabla \cdot\left(\boldsymbol{\tau}-I^{h} \boldsymbol{\tau}\right)\right\|_{0, \beta} \leq C h^{m_{2}}\left(|\nabla \cdot \boldsymbol{\tau}|_{1, \beta}+|\nabla \cdot \boldsymbol{\tau}|_{\alpha}\right),
$$

where $m_{1}=\min \{1, \beta+\tilde{\alpha}\}, m_{2}=\min \{1, \beta+\alpha\}$, and $C$ depends only on $k, \beta$, and the shape of $\Omega$.

Proof. We first prove the bound in (3.8). Note that (3.4) and (3.5) apply since $\boldsymbol{\tau}$ is smooth enough to be in the domain of $I^{h}$. Denote the set of elements adjacent to $\mathcal{E}$ by

$$
\mathcal{K}_{0}=\left\{K \in \mathcal{T}_{h}: \bar{K} \cap \mathcal{E} \neq \emptyset\right\} .
$$

For any element $K \in \mathcal{T}_{h} \backslash \mathcal{K}_{0}$, we note that $\tau \in H^{1}(K)$ and that

$$
\frac{1}{c_{K}} h_{K} \leq r_{\min } \leq r \leq r_{\max } \leq r_{\min }+c_{K} h_{K}
$$

where $r_{\min }$ and $r_{\max }$ are the minimum and maximum distances from $K$ to $\mathcal{E}$, respectively, and $c_{K}$ depends only on $d$ and the shape of $K$ (e.g., $c_{K}=\sqrt{d}$ for a uniform mesh). (3.10) implies

$$
\frac{r_{\max }}{r_{\min }} \leq 1+c_{K}^{2}
$$

Now, it follows from (3.10) and (3.4) with $m=1$ and $s=0$ that

$$
\begin{aligned}
\left\|\boldsymbol{\tau}-I^{h} \boldsymbol{\tau}\right\|_{0, \beta ; K} & =\left\|r^{\beta}\left(\boldsymbol{\tau}-I^{h} \boldsymbol{\tau}\right)\right\|_{0 ; K} \leq r_{\max }^{\beta}\left\|\boldsymbol{\tau}-I^{h} \boldsymbol{\tau}\right\|_{0 ; K} \\
& \leq C r_{\max }^{\beta} h_{K}|\boldsymbol{\tau}|_{1 ; K} \leq C r_{\max }^{\beta} h_{K}\left\|\left(r / r_{\min }\right)^{\beta} \nabla \boldsymbol{\tau}\right\|_{0 ; K} \\
& =C\left(\frac{r_{\max }}{r_{\min }}\right)^{\beta} h_{K}|\boldsymbol{\tau}|_{1, \beta ; K}=C h_{K}|\boldsymbol{\tau}|_{1, \beta ; K} .
\end{aligned}
$$

For any element $K \in \mathcal{K}_{0}$, it follows from the fact that $r \leq h_{K}$ and (3.4) that

$$
\begin{aligned}
\left\|\boldsymbol{\tau}-I^{h} \boldsymbol{\tau}\right\|_{0, \beta ; K} & =\left\|r^{\beta}\left(\boldsymbol{\tau}-I^{h} \boldsymbol{\tau}\right)\right\|_{0 ; K} \leq h_{K}^{\beta}\left\|\boldsymbol{\tau}-I^{h} \boldsymbol{\tau}\right\|_{0 ; K} \\
& \leq C h_{K}^{\beta+\tilde{\alpha}}|\boldsymbol{\tau}|_{\alpha ; K},
\end{aligned}
$$

which, together with (3.11), yields the bound in (3.8).

To prove (3.9), we first consider $K \in \mathcal{T}_{h} \backslash \mathcal{K}_{0}$. A calculation identical to (3.11) using (3.5) with $m=1$ gives

$$
\left\|\nabla \cdot\left(\boldsymbol{\tau}-I^{h} \boldsymbol{\tau}\right)\right\|_{0, \beta ; K} \leq C h_{K}|\nabla \cdot \boldsymbol{\tau}|_{1, \beta ; K} .
$$

Now consider $K \in \mathcal{K}_{0}$. Let $\Pi^{h}$ be the $L^{2}$-projection onto discontinuous piecewise polynomial of degree $k$. Working with affine elements allows the use of the wellknown commutativity property,

$$
\nabla \cdot\left(I^{h} \boldsymbol{\tau}\right)=\Pi^{h} \nabla \cdot \boldsymbol{\tau}
$$

This, (3.5), and the approximation property of $\Pi^{h}$ gives

$$
\begin{aligned}
\left\|\nabla \cdot\left(\boldsymbol{\tau}-I^{h} \boldsymbol{\tau}\right)\right\|_{0, \beta ; K} & =\left\|\left(I-\Pi^{h}\right) \nabla \cdot \boldsymbol{\tau}\right\|_{0, \beta ; K} \\
& \leq h_{K}^{\beta}\left\|\left(I-\Pi^{h}\right) \nabla \cdot \boldsymbol{\tau}\right\|_{0 ; K} \\
& \leq C h_{K}^{\beta+\alpha}|\nabla \cdot \boldsymbol{\tau}|_{\alpha ; K} .
\end{aligned}
$$


This and (3.12) leads to the bound in (3.9). The proof of the theorem is now completed.

Lemma 3.3 If $\beta \leq \gamma$, then

$$
\|\phi\|_{0, \gamma} \leq C\|\phi\|_{0, \beta} \quad \forall \phi \in H_{\beta}^{0}(\Omega) .
$$

Proof. Let $\Omega_{1}=\Omega \cap\{r<1\}$. For $r<1$, since $r^{\gamma} \leq r^{\beta}$, we then have

$$
\|\phi\|_{0, \gamma ; \Omega_{1}}=\left\|r^{\gamma} \phi\right\|_{0 ; \Omega_{1}} \leq\left\|r^{\beta} \phi\right\|_{0 ; \Omega_{1}}=\|\phi\|_{0, \beta ; \Omega_{1}} .
$$

Now, (3.13) is a direct consequence of the fact that $\|\phi\|_{0, \gamma ; \Omega_{2}} \leq C\|\phi\|_{0, \beta ; \Omega_{2}}$, where $\Omega_{2}=\Omega \backslash \bar{\Omega}_{1}$.

The following theorem establishes equivalence of $G(u, \boldsymbol{\sigma} ; 0)^{1 / 2}$ to the norm

$$
\|(u, \boldsymbol{\sigma})\|_{\beta_{1}, \beta_{2}}:=\left(\|u\|_{1, \beta_{1}}^{2}+\|\boldsymbol{\sigma}\|_{0, \beta_{1}}^{2}+\|\nabla \cdot \boldsymbol{\sigma}\|_{0, \beta_{2}}^{2}\right)^{1 / 2} .
$$

Since $G\left(u^{h}, \boldsymbol{\sigma}^{h} ; f\right)=G\left(u-u^{h}, \boldsymbol{\sigma}-\boldsymbol{\sigma}^{h} ; 0\right)$, this equivalence implies that minimizing the functional is equivalent to minimizing the error in this natural norm.

Theorem 3.4 For $\beta_{1} \leq \beta_{2} \leq \beta_{1}+1$ and $\left|\beta_{1}\right|<\alpha$, there exist positive constants $c_{0}$ and $c_{1}$ such that

$$
c_{0}\|(u, \boldsymbol{\sigma})\|_{\beta_{1}, \beta_{2}}^{2} \leq G(u, \boldsymbol{\sigma} ; 0) \leq c_{1}\|(u, \boldsymbol{\sigma})\|_{\beta_{1}, \beta_{2}}^{2}
$$

for all $(u, \sigma) \in \mathcal{V} \times \mathcal{W}$.

Proof. We first recall the definition of the homogeneous functional

$$
G(u, \boldsymbol{\sigma} ; 0)=\left\|A^{-1 / 2}(\boldsymbol{\sigma}+A \nabla u)\right\|_{0, \beta_{1}}^{2}+\|\nabla \cdot \boldsymbol{\sigma}+X u\|_{0, \beta_{2}}^{2} .
$$

Since $\beta_{1}-1<\beta_{1} \leq \beta_{2}$, (3.13) with the respective $\phi=\nabla u, \beta=\beta_{1}, \gamma=\beta_{2}$ and $\phi=u$, $\beta=\beta_{1}-1, \gamma=\beta_{2}$ implies

$$
\|\nabla u\|_{0, \beta_{2}}+\|u\|_{0, \beta_{2}} \leq C\left(\|\nabla u\|_{0, \beta_{1}}+\|u\|_{0, \beta_{1}-1}\right)=C\|u\|_{1, \beta_{1}} .
$$

These, together with the triangle inequality and the assumptions on $A$ and $X$, proves the validity of the upper bound in (3.14).

For the lower bound in (3.14), since $\left|\beta_{1}\right|<\alpha$, Theorem 3.1 with $\beta=\beta_{1}$ and the triangle inequality gives

$$
\begin{aligned}
\|u\|_{1, \beta_{1}} & \leq C\|-\nabla \cdot A \nabla u+X u\|_{-1, \beta_{1}} \\
& \leq C\left(\|\nabla \cdot \boldsymbol{\sigma}+\nabla \cdot A \nabla u\|_{-1, \beta_{1}}+\|\nabla \cdot \boldsymbol{\sigma}+X u\|_{-1, \beta_{1}}\right) .
\end{aligned}
$$

We consider each term on the right side above separately. For the first term, integration by parts and the Cauchy-Schwarz inequality give

$$
\begin{aligned}
\|\nabla \cdot \boldsymbol{\sigma}+\nabla \cdot A \nabla u\|_{-1, \beta_{1}} & =\sup _{\phi \in D_{-\beta_{1}}^{1}} \frac{\langle\nabla \cdot(\boldsymbol{\sigma}+A \nabla u), \phi\rangle}{\|\phi\|_{1,-\beta_{1}}} \\
& =\sup _{\phi \in D_{-\beta_{1}}^{1}} \frac{\left\langle-r^{\beta_{1}}(\boldsymbol{\sigma}+A \nabla u), r^{-\beta_{1}} \nabla \phi\right\rangle}{\|\phi\|_{1,-\beta_{1}}} \\
& \leq\left\|r^{\beta_{1}}(\boldsymbol{\sigma}+A \nabla u)\right\| \sup _{\phi \in D_{-\beta_{1}}^{1}} \frac{\|\nabla \phi\|_{0,-\beta_{1}}}{\|\phi\|_{1,-\beta_{1}}} \\
& \leq\|\boldsymbol{\sigma}+A \nabla u\|_{0, \beta_{1}} .
\end{aligned}
$$


Since $\beta_{2} \leq \beta_{1}+1,(3.13)$ with $\beta=-\left(\beta_{1}+1\right)$ and $\gamma=-\beta_{2}$ gives

$$
\|\phi\|_{0,-\beta_{2}} \leq C\|\phi\|_{0,-\left(\beta_{1}+1\right)}=C\left\|r^{-\beta_{1}-1} \phi\right\| \leq C\|\phi\|_{1,-\beta_{1}} .
$$

Now, the second term may be bounded similarly using the Cauchy-Schwarz inequality and (3.18),

$$
\begin{aligned}
\|\nabla \cdot \boldsymbol{\sigma}+X u\|_{-1, \beta_{1}} & =\sup _{\phi \in D_{-\beta_{1}}^{1}} \frac{\langle\nabla \cdot \boldsymbol{\sigma}+X u, \phi\rangle}{\|\phi\|_{1,-\beta_{1}}} \\
& =\sup _{\phi \in D_{-\beta_{1}}^{1}} \frac{\left\langle r^{\beta_{2}}(\nabla \cdot \boldsymbol{\sigma}+X u), r^{-\beta_{2}} \phi\right\rangle}{\|\phi\|_{1,-\beta_{1}}} \\
& \leq\|\nabla \cdot \boldsymbol{\sigma}+X u\|_{0, \beta_{2}} \sup _{\phi \in D_{-\beta_{1}}^{1}} \frac{\|\phi\|_{0,-\beta_{2}}}{\|\phi\|_{1,-\beta_{1}}} \\
& \leq C\|\nabla \cdot \boldsymbol{\sigma}+X u\|_{0, \beta_{2}} .
\end{aligned}
$$

Combining equations (3.16), (3.17), and (3.19) give

$$
\|u\|_{1, \beta_{1}} \leq C G(u, \boldsymbol{\sigma} ; 0)^{1 / 2} .
$$

It follows from the triangle inequality, (3.1), (3.20), and (3.15) that

$$
\begin{aligned}
& \|\boldsymbol{\sigma}\|_{0, \beta_{1}}+\|\nabla \cdot \boldsymbol{\sigma}\|_{0, \beta_{2}} \\
\leq & \|\boldsymbol{\sigma}+A \nabla u\|_{0, \beta_{1}}+\|A \nabla u\|_{0, \beta_{1}}+\|\nabla \cdot \boldsymbol{\sigma}+X u\|_{0, \beta_{2}}+\|X u\|_{0, \beta_{2}} \\
\leq & C G(u, \boldsymbol{\sigma} ; 0)^{1 / 2}+C\|u\|_{1, \beta_{1}}+C\left(\|\nabla u\|_{0, \beta_{2}}+\|u\|_{0, \beta_{2}}\right) \\
\leq & C G(u, \boldsymbol{\sigma} ; 0)^{1 / 2}
\end{aligned}
$$

which, together with (3.20), implies the lower bound in (3.14) and, hence, completes the proof of the theorem.

We may now prove error estimates.

Theorem 3.5 Let $(u, \boldsymbol{\sigma}) \in \mathcal{V} \times \mathcal{W}$ be the solution of system (2.1) and $\left(u^{h}, \boldsymbol{\sigma}^{h}\right)$ be the minimizer of the weighted functional (2.2) over $\mathcal{V}^{h} \times \mathcal{W}^{h}$. Assume that $f \in H^{\alpha}(\Omega)$. Then for $\beta_{1} \in[1-\alpha, \alpha)$ and $\beta_{2} \in\left[\beta_{1}, \beta_{1}+1\right]$, the following error estimate holds

$$
\left\|\left(u-u^{h}, \boldsymbol{\sigma}-\boldsymbol{\sigma}^{h}\right)\right\|_{\beta_{1}, \beta_{2}} \leq C h^{m_{1}}\left(\|u\|_{2, \beta_{1}}+\|u\|_{1+\alpha}+\|f\|_{\alpha}\right)
$$

with $m_{1}=\min \left\{1, \beta_{1}+\tilde{\alpha}\right\}$.

Proof. For any $(v, \boldsymbol{\tau}) \in \mathcal{V}^{h} \times \mathcal{W}^{h}$, it follows from Theorem 3.4 and the orthogonality property that

$$
\begin{aligned}
c_{0}\left\|\left(u-u^{h}, \boldsymbol{\sigma}-\boldsymbol{\sigma}^{h}\right)\right\|_{\beta_{1}, \beta_{2}}^{2} \leq G\left(u-u^{h}, \boldsymbol{\sigma}-\boldsymbol{\sigma}^{h} ; 0\right) \\
\quad=G\left(u-v^{h}, \boldsymbol{\sigma}-\boldsymbol{\tau}^{h} ; 0\right) \leq c_{1}\left\|\left(u-v^{h}, \boldsymbol{\sigma}-\boldsymbol{\tau}^{h}\right)\right\|_{\beta_{1}, \beta_{2}}^{2},
\end{aligned}
$$


which implies

$$
\begin{aligned}
& \left\|\left(u-u^{h}, \boldsymbol{\sigma}-\boldsymbol{\sigma}^{h}\right)\right\|_{\beta_{1}, \beta_{2}}^{2} \leq \frac{c_{1}}{c_{0}} \inf _{(v, \boldsymbol{\tau}) \in \mathcal{V}^{h} \times \mathcal{W}^{h}}\|(u-v, \boldsymbol{\sigma}-\boldsymbol{\tau})\|_{\beta_{1}, \beta_{2}}^{2} \\
& \quad \leq C\left(\inf _{v \in \mathcal{V}}\|u-v\|_{1, \beta_{1}}^{2}+\inf _{\boldsymbol{\tau} \in \mathcal{W}^{h}}\|\boldsymbol{\sigma}-\boldsymbol{\tau}\|_{0, \beta_{1}}^{2}+\inf _{\boldsymbol{\tau} \in \mathcal{W}^{h}}\|\nabla \cdot(\boldsymbol{\sigma}-\boldsymbol{\tau})\|_{0, \beta_{2}}^{2}\right) .
\end{aligned}
$$

This, with the approximation properties in (3.3), (3.8), and (3.9) results in

$$
\left\|\left(u-u^{h}, \boldsymbol{\sigma}-\boldsymbol{\sigma}^{h}\right)\right\|_{\beta_{1}, \beta_{2}}^{2} \leq C h^{2 m_{1}}\left(\|u\|_{2, \beta_{1}}^{2}+|\boldsymbol{\sigma}|_{1, \beta_{1}}^{2}+|\boldsymbol{\sigma}|_{\alpha}^{2}+|\nabla \cdot \boldsymbol{\sigma}|_{\alpha}^{2}\right) .
$$

Choosing $\beta_{1} \geq 1-\alpha$ ensures that $u \in H_{\beta_{1}}^{2}(\Omega)$. The terms above involving $\sigma$ each may be bounded as follows:

$$
\begin{gathered}
|\boldsymbol{\sigma}|_{1, \beta_{1}}=|A \nabla u|_{1, \beta_{1}} \leq C\|u\|_{2, \beta_{1}}, \\
|\boldsymbol{\sigma}|_{\alpha}=|A \nabla u|_{\alpha} \leq C\|u\|_{1+\alpha}, \\
|\nabla \cdot \boldsymbol{\sigma}|_{\alpha}=|f-X u|_{\alpha} \leq|f|_{\alpha}+|X u|_{\alpha} \leq|f|_{\alpha}+C\|u\|_{1+\alpha},
\end{gathered}
$$

and the theorem follows by combining these bounds above.

Theorem 3.5 shows improvement in the order of approximation by choosing $\beta_{1} \in(0, \alpha]$. We note that Theorem 3.5 assumes $\beta_{1} \geq 1-\alpha$ to be consistent with interpolation bound (3.3). In practice this restriction is unnecessary. Choosing $\beta_{1}=1-\alpha$ restores exactly $O(h)$ convergence. Since for many elliptic problems (Poisson, Stokes, linear elasticity, etc.) we have $\alpha \in[0.5,1)$, we may choose $\beta_{1} \in[1-\alpha, \alpha)$ or simply $\beta_{1}=0.5$. This flexibility in parameter choice shows that almost no a priori information of the singular solutions is required for this approach to apply. Rough lower bounds on $\alpha$ suffice.

We also may choose to apply the techniques here locally to each singular point in the domain. The loss of smoothness of the solution at irregular boundary points is a local phenomenon, but it causes global difficulties. Our motivation is to provide a local treatment that eliminates the global effect of the irregular boundary point. To this end, let $\Omega_{R}=\{\mathbf{x} \in \Omega: r>R\}$ be a subdomain of $\Omega$ of fixed size that does not include $\mathcal{E}$. Since on $\Omega_{R}$ the norms $\|(u, \boldsymbol{\sigma})\|_{0,0}$ and $\|(u, \boldsymbol{\sigma})\|_{\beta_{1}, \beta_{2}}$ are equivalent, Theorem 3.5 indicates that the error is reduced at optimal asymptotic rates away from $\mathcal{E}$-thus eliminating the pollution effect.

We conclude this section with a brief justification of the reduction of the error with respect to a non-weighted norm.

Theorem 3.6 Let $\beta_{1} \geq 1-\tilde{\alpha}$. Then under the same assumptions as Theorem 3.5, the error in approximating $\boldsymbol{\sigma}$ satisfies

$$
\left\|\boldsymbol{\sigma}-\boldsymbol{\sigma}^{h}\right\| \leq C h^{\tilde{\alpha}}
$$

where $C$ is independent of $h$.

Proof. By the triangle inequality we take

$$
\left\|\boldsymbol{\sigma}-\boldsymbol{\sigma}^{h}\right\| \leq\left\|\boldsymbol{\sigma}-I^{h} \boldsymbol{\sigma}\right\|+\left\|\boldsymbol{\sigma}^{h}-I^{h} \boldsymbol{\sigma}\right\|
$$


and consider the terms above separately. The first term may be bounded by (3.6),

$$
\left\|\boldsymbol{\sigma}-I^{h} \boldsymbol{\sigma}\right\| \leq C h^{\tilde{\alpha}}|\boldsymbol{\sigma}|_{\alpha} .
$$

For the second term, we require an inequality proved in [10]:

$$
\left\|\boldsymbol{\tau}^{h}\right\| \leq C h^{-\delta}\left\|\boldsymbol{\tau}^{h}\right\|_{0, \delta}
$$

for $\delta>0$ and all $\boldsymbol{\tau}^{h} \in \mathcal{W}^{h}$. Now, using (3.24), the triangle inequality, Theorem 3.5 and (3.8) results in

$$
\begin{aligned}
\left\|\boldsymbol{\sigma}^{h}-I^{h} \boldsymbol{\sigma}\right\| & \leq C h^{-\beta_{1}}\left\|\boldsymbol{\sigma}^{h}-I^{h} \boldsymbol{\sigma}\right\|_{0, \beta_{1}} \\
& \leq C h^{-\beta_{1}}\left(\left\|\boldsymbol{\sigma}-\boldsymbol{\sigma}^{h}\right\|_{0, \beta_{1}}+\left\|\boldsymbol{\sigma}-I^{h} \boldsymbol{\sigma}\right\|_{0, \beta_{1}}\right) \\
& \leq C h^{-\beta_{1}} h^{\beta_{1}+\tilde{\alpha}} \leq C h^{\tilde{\alpha}} .
\end{aligned}
$$

Combining (3.22), (3.23) and (3.25) yields the bound in (3.21), and thus completes the theorem.

4. Numerical Results. In this section we validate the weighted norm method by presenting two numerical test problems. Define the domain $\Omega=\{(r, \theta): r<1, \theta \in$ $(0,7 \pi / 4)\}$ as pictured in figure 4.1 , which results in singularities with $\alpha=4 / 7$.

While the analysis in this paper assumes one singular corner with norms weighted by $r^{\beta_{1}}$ and $r^{\beta_{2}}$, generalization to more than one singular corner is straightforward. We illustrate that here by defining the local weight functions

$$
w_{i}=\left\{\begin{aligned}
(r / R)^{\beta_{i}} & \text { for } r<R \\
1 & \text { for } r \geq R
\end{aligned}\right.
$$

for $i=1,2$ and $R=0.25$. We minimize the weighted functional

$$
G(u, \boldsymbol{\sigma} ; f)=\left\|w_{1} A^{-1 / 2}(\boldsymbol{\sigma}+A \nabla u)\right\|^{2}+\left\|w_{2}(\nabla \cdot \boldsymbol{\sigma}+\mathbf{b} \cdot \nabla u+c u-f)\right\|^{2},
$$

for $u^{h}$ using continuous $P_{1}$ finite elements and $\boldsymbol{\sigma}^{h}$ using $R T_{0}$ elements. We take $\beta_{1}=\beta_{2}=0.57 \approx \alpha$ as the power of the weight functions. A regular triangulation of the domain is used, where the meshsize parameter is $h=\min _{K \in \mathcal{T}^{h}} \operatorname{diam}(K)$.
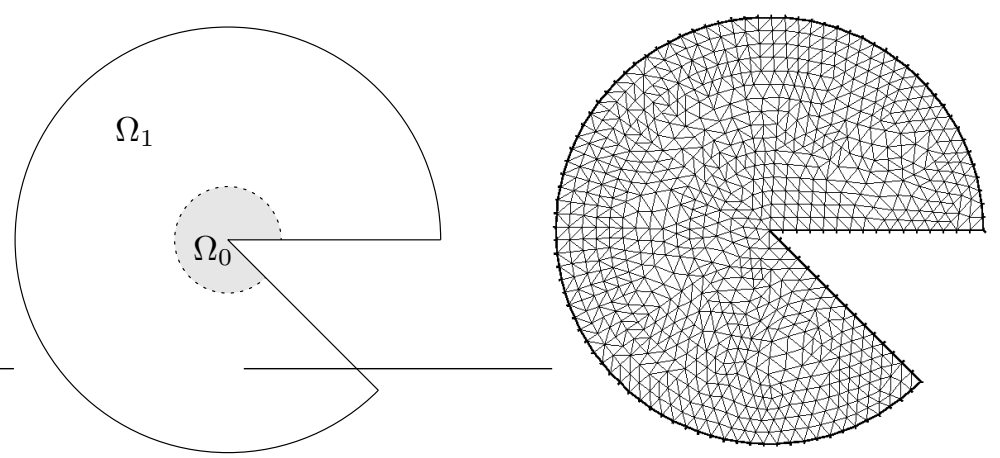

FIG. 4.1. Test domain $\Omega$, partitioned into subdomains $\Omega_{0}$ and $\Omega_{1}$, and the initial discretization, $M_{1}$

Solving on the sequence of discretizations described in table 4.1, we compare the computed convergence rate from the two finest meshes and compare to the asymptotic 


$\mid$\begin{tabular}{|c|r|c|}
\hline mesh & \# elements & $h$ \\
\hline$M_{1}$ & 1576 & $5.72 \mathrm{e}-2$ \\
\hline$M_{2}$ & 6304 & $2.86 \mathrm{e}-2$ \\
\hline$M_{3}$ & 25216 & $1.43 \mathrm{e}-2$ \\
\hline$M_{4}$ & 100864 & $7.15 \mathrm{e}-3$ \\
\hline
\end{tabular}
Computational meshes used for both example problems.

rate that the interpolant admits (e.g., see equations (3.2) and (3.4)). We refer to this as the asymptotic optimal convergence rate (i.e., asy. opt. in table 4.2).

We denote the exact solution by $u$, strongly impose Dirichlet boundary conditions on $\partial \Omega$, and compute the right side function $f$ by analytically computing

$$
f=-\nabla \cdot A \nabla u+\mathbf{b} \cdot \nabla u+c u .
$$

We also analytically define $\boldsymbol{\sigma}=-A \nabla u$ for comparison to the numerical approximation, $\sigma^{h}$.

Problem 1: Poisson's Equation. As a baseline case we begin with the Laplace operator,

$$
A=\left(\begin{array}{ll}
1 & 0 \\
0 & 1
\end{array}\right), \quad \mathbf{b}=\left(\begin{array}{l}
0 \\
0
\end{array}\right), \quad \text { and } \quad c=0 .
$$

The exact solution is chosen to be $u=r^{\alpha} \sin (\alpha \theta)$, and in this case $f=0$.

Problem 2: Convection Dominated Anisotropic Diffusion. We now consider an operator consisting of anisotropic diffusion dominated by a strong convection with a small reaction term,

$$
A=\left(\begin{array}{cc}
0.1 & 0 \\
0 & 1
\end{array}\right), \quad \mathbf{b}=\left(\begin{array}{c}
10 \\
5
\end{array}\right), \quad \text { and } \quad c=1
$$

The exact solution in this case is chosen to be $u=\tilde{r}^{\alpha} \sin (\alpha \tilde{\theta})$, where $(\tilde{r}, \tilde{\theta})$ are polar coordinates in the rescaled domain defined by $\left(\tilde{x}_{1}, \tilde{x}_{2}\right)^{t}=A^{-1 / 2}\left(x_{1}, x_{2}\right)^{t}$. The resulting data function satisfies $f \in H^{k}(\Omega)$ for any $k<\alpha$ and takes the form

$$
f=10 \alpha \tilde{r}^{\alpha-1} \sin ((\alpha-1) \tilde{\theta})+5 \alpha \tilde{r}^{\alpha-1} \cos ((\alpha-1) \tilde{\theta})+\tilde{r}^{\alpha} \sin \alpha \tilde{\theta} .
$$

To distinguish the rates of convergence close to and far from the singular point we define the following partitioning of the domain: $\Omega_{0}=\{r<1 / 4\} \cap \Omega$ and $\Omega_{1}=\Omega \backslash \Omega_{0}$. Thus, $\Omega_{0}$ contains the singular point and $\Omega_{1}$ does not. We solve the problem in $\Omega$, but monitor the error in these subdomains as measured in the functional norm, $G^{1 / 2}=G\left(u^{h}, \sigma^{h} ; f\right)^{1 / 2}$, the $L^{2}$ errors in $\sigma$, and the $H^{1}$ seminorm error in $u$.

Table 4.2 summarizes the computational results of the two problems. In both problems, the weighted functional is reduced at (or very nearly at) optimal $O(h)$, as predicted by Theorem 3.5. This rate is seen on both partitions of the domain. For both $\left\|\boldsymbol{\sigma}-\boldsymbol{\sigma}^{h}\right\|$ and $\left|u-u^{h}\right|_{1}$, the optimal rates are $O\left(h^{4 / 7}\right)$ and $O(h)$ in $\Omega_{0}$ and $\Omega_{1}$ respectively. Both example problems have similar behavior, though example 1 approaches the asymptotic rate faster than the more challenging example 2 . The 
Problem 1

Problem 2

\begin{tabular}{|r||c|c|c||c|c|c|}
\hline$G^{1 / 2}$ & $\Omega$ & $\Omega_{0}$ & $\Omega_{1}$ & $\Omega$ & $\Omega_{0}$ & $\Omega_{1}$ \\
\hline mesh $M_{1}$ & $8.57 \mathrm{e}-2$ & $7.65 \mathrm{e}-2$ & $3.84 \mathrm{e}-2$ & $8.94 \mathrm{e}-1$ & $7.45 \mathrm{e}-1$ & $4.94 \mathrm{e}-1$ \\
$M_{2}$ & $4.30 \mathrm{e}-2$ & $3.85 \mathrm{e}-2$ & $1.91 \mathrm{e}-2$ & $4.67 \mathrm{e}-1$ & $3.94 \mathrm{e}-1$ & $2.49 \mathrm{e}-1$ \\
$M_{3}$ & $2.15 \mathrm{e}-2$ & $1.93 \mathrm{e}-2$ & $9.52 \mathrm{e}-3$ & $2.40 \mathrm{e}-1$ & $2.05 \mathrm{e}-1$ & $1.25 \mathrm{e}-1$ \\
$M_{4}$ & $1.08 \mathrm{e}-2$ & $9.66 \mathrm{e}-3$ & $4.75 \mathrm{e}-3$ & $1.23 \mathrm{e}-1$ & $1.06 \mathrm{e}-2$ & $6.23 \mathrm{e}-2$ \\
\hline conv. rate & 1.00 & 1.00 & 1.00 & 0.98 & 0.96 & 1.00 \\
asy. opt. & 1 & 1 & 1 & 1 & 1 & 1 \\
\hline \hline $\boldsymbol{\sigma}-\sigma^{h} \|$ & $\Omega$ & $\Omega_{0}$ & $\Omega_{1}$ & $\Omega$ & $\Omega_{0}$ & $\Omega_{1}$ \\
\hline mesh $M_{1}$ & $1.19 \mathrm{e}-1$ & $1.16 \mathrm{e}-1$ & $2.84 \mathrm{e}-2$ & $8.60 \mathrm{e}-2$ & $8.12 \mathrm{e}-2$ & $2.84 \mathrm{e}-2$ \\
$M_{2}$ & $8.15 \mathrm{e}-2$ & $8.03 \mathrm{e}-2$ & $1.39 \mathrm{e}-2$ & $5.21 \mathrm{e}-2$ & $5.06 \mathrm{e}-2$ & $1.26 \mathrm{e}-2$ \\
$M_{3}$ & $5.52 \mathrm{e}-2$ & $5.48 \mathrm{e}-2$ & $6.93 \mathrm{e}-3$ & $3.21 \mathrm{e}-2$ & $3.16 \mathrm{e}-2$ & $5.84 \mathrm{e}-3$ \\
$M_{4}$ & $3.73 \mathrm{e}-2$ & $3.72 \mathrm{e}-2$ & $3.46 \mathrm{e}-3$ & $2.03 \mathrm{e}-2$ & $2.02 \mathrm{e}-2$ & $2.79 \mathrm{e}-3$ \\
\hline conv. rate & 0.57 & 0.56 & 1.00 & 0.61 & 0.60 & 1.05 \\
asy. opt. & $4 / 7$ & $4 / 7$ & 1 & $4 / 7$ & $4 / 7$ & 1 \\
\hline \hline$\left|u-u^{h}\right|_{1}$ & $\Omega$ & $\Omega_{0}$ & $\Omega_{1}$ & $\Omega$ & $\Omega_{0}$ & $\Omega_{1}$ \\
\hline mesh $M_{1}$ & $1.10 \mathrm{e}-1$ & $1.07 \mathrm{e}-1$ & $2.58 \mathrm{e}-2$ & $2.51 \mathrm{e}-1$ & $2.41 \mathrm{e}-1$ & $7.17 \mathrm{e}-2$ \\
$M_{2}$ & $7.42 \mathrm{e}-2$ & $7.31 \mathrm{e}-2$ & $1.30 \mathrm{e}-2$ & $1.66 \mathrm{e}-1$ & $1.62 \mathrm{e}-1$ & $3.56 \mathrm{e}-2$ \\
$M_{3}$ & $4.99 \mathrm{e}-2$ & $4.95 \mathrm{e}-2$ & $6.51 \mathrm{e}-3$ & $1.09 \mathrm{e}-1$ & $1.07 \mathrm{e}-1$ & $1.75 \mathrm{e}-2$ \\
$M_{4}$ & $3.36 \mathrm{e}-2$ & $3.34 \mathrm{e}-2$ & $3.26 \mathrm{e}-3$ & $7.12 \mathrm{e}-2$ & $7.07 \mathrm{e}-2$ & $8.66 \mathrm{e}-3$ \\
\hline conv. rate & 0.57 & 0.57 & 1.00 & 0.61 & 0.60 & 1.02 \\
asy. opt. & $4 / 7$ & $4 / 7$ & 1 & $4 / 7$ & $4 / 7$ & 1 \\
\hline
\end{tabular}

Numerical results for Problems 1 and 2.

results also show no pollution effect as evidenced by a clear distinction in convergence rates between partitions $\Omega_{0}$ and $\Omega_{1}$.

We also note that in (3.6) and (3.7) we assume $\tilde{\alpha} \in(0, \alpha]$. The rates we achieve in table 4.2 indicate that $\tilde{\alpha}=\alpha$. We also observe this same result in many numerical tests not shown here.

\section{REFERENCES}

[1] C. BaCUTA, V. Nistor, And L. Zikatanov, Improving the rate of convergence of high-order finite elements on polyhedra $i$ : a priori estimates, Numer. Funct. Anal. Optim., 26 (2005), pp. 613-639.

[2] D. Braess, Finite Elements: Theory, Fast Solvers and Applications in Solid Mechanics, Cambridge, 2001.

[3] F. Brezzi And M. Fortin, Mixed and Hybrid Finite Element Methods, Springer-Verlag, 1991.

[4] Z. CAI AND J. KU, The $L^{2}$ norm error estimates for the div least-squares method, SIAM J. Numer. Anal., 44 (2006), pp. 1721-1734.

[5] Z. Cai, R. Lazarov, T. Manteuffel, and S. MCCormick, First-order system least squares for second-order partial differential equations: part i, SIAM J. Numer. Anal., 31 (1994), pp. 1785-1799.

[6] C. COX AND G. FIX, On the accuracy of least squares methods in the presence of corner singularities, Comp. and Maths. with Appls., 10 (1984), pp. 463-475.

[7] P. Grisvard, Singularities in Boundary Value Problems, Springer-Verlag, 1992. 
[8] V. Kondratiev, Boundary problems for elliptic equations in domains with conical or angular points, Trans. Moscow Math. Soc., 16 (1967), pp. 227-313.

[9] J. KU, A remark on the coercivity for a first-order least-squares method, Numer. Methods PDEs. To Appear.

[10] E. Lee, T. Manteuffel, and C. Westphal, Weighted-norm first-order system least squares (fosls) for problems with corner singularities, SIAM J. Numer. Anal., 44 (2006), pp. 19741996.

[11] - Weighted-norm first-order system least squares (fosls) for div/curl systems with three dimensional edge singularities, SIAM J. Numer. Anal., (to appear).

[12] S. NiCAISE, Regularity of the solutions of elliptic systems in polyhedral domains, Bull. Belg. Math. Soc., 4 (1997), pp. 411-429. 